\title{
Source memory for unrecognized items: Predictions from multivariate signal detection theory
}

\author{
Jeffrey J. Starns, Jason L. Hicks, Noelle L. Brown, and Benjamin A. Martin \\ Louisiana State University, Baton Rouge, Louisiana
}

\begin{abstract}
We report three experiments investigating source memory for words that were called "new" on a recognition test. In each experiment, participants could accurately specify the source of words that they failed to recognize. Results also demonstrated that source memory for unrecognized items varied with the bias to respond "old" in recognition decisions: Participants displayed unrecognized source memory when they were told that $25 \%$ of the recognition test words were old (promoting conservative responding) but not when they were told that $75 \%$ of the test words were old (promoting liberal responding). Our results were successfully predicted by a multivariate signal detection approach to recognition/source memory.
\end{abstract}

Memory researchers distinguish between the ability to recognize whether an item was previously encountered and the ability to specify the presentation context or source of the item. Although recognition and source retrieval can be considered separate forms of memory, they are typically tested simultaneously, and many models are designed to simultaneously accommodate recognition and source data-for example, multinomial models (Batchelder \& Riefer, 1990) and multivariate signal detection models (DeCarlo, 2003). These integrative theoretical perspectives help to reveal underlying processes that are common to both recognition and source retrieval, thereby allowing memory researchers to gain insight into the relationship between these expressions of memory.

In the present study, we investigated one aspect of the source/recognition relationship - namely, whether or not people have the ability to accurately remember the source characteristics of items that they failed to recognize. The test procedures typically used by source memory researchers reflect the assumption that participants have no ability to discriminate sources in the absence of recognition memory. For example, when source decisions follow recognition decisions, the standard practice is to require source responses only for items receiving positive recognition responses. Another common test procedure is to have participants select "Source A," "Source B," or "new" for each test item, in which case there is no way for participants to claim a particular source without also claiming that the item was studied (i.e., that it is not new). In the present study, we required source decisions for unrecognized items. In the following paragraphs, we describe multinomial models and note that they were developed on the basis of the assumption that source discrimination is not possible for unrecognized items. We also discuss multivariate signal detection models and demonstrate that these models suggest that source discrimination is possible for unrecognized items in certain circumstances.

Multinomial models have been extensively applied in the source memory literature (see, e.g., Bayen, Murnane, \& Erdfelder, 1996; Dodson, Holland, \& Shimamura, 1998; Dodson \& Shimamura, 2000; Meiser \& Bröder, 2002), and existing multinomial models formalize the assumption that source discrimination can be achieved only for recognized items. Multinomial models analyze recognition and source memory in terms of underlying cognitive states that are experienced in a discrete fashion (Batchelder \& Riefer, 1990). Each model parameter estimates the probability of experiencing a certain cognitive state when a certain type of item is presented as a test candidate. Figure 1 displays a representative multinomial model for a recognition/source test with three classes of items (Source A, Source B, and new). The item classes appear on the left, the available responses appear on the right, and in between is a processing tree structure representing how inferred underlying states lead to particular responses.

Inspecting the model structure for Source A and Source B items reveals the cognitive states that can be experienced for studied items. With a probability measured by the $D$ parameters, participants detect that items from Source A or Source B were studied. In the absence of item detection $(1-D)$, participants must guess the test word's presentation status. Participants guess that the word was studied with a probability measured by the $b$ parameter. The $d$ parameters represent the probability of identifying the source of the test item. In the absence of source identification, participants guess a source. The $a$ parameter rep- 
resents the probability of guessing "Source A" for items that inspired item detection but not source identification, and the $g$ parameter represents the probability of guessing "Source A" for items that participants claimed to have studied on the basis of guessing.

The multinomial model in Figure 1 clearly demonstrates that source identification is conditional on successful item detection. For both sources, the source identification parameter $(d)$ appears only in conjunction with the item detection parameter $(D)$. When participants must make a source decision for an item that is not detected, they can only guess. This assumption of the model is clearly revealed in the underlying states proposed for words that were not detected as studied but were called "studied" through guessing [along the processing tree paths $(1-D) * b$ ]. The only parameter determining source responses for these items is a guessing parameter $(g)$. Although Figure 1 shows only a single representative model, this property is shared by all of the multinomial models that have been previously applied to source memory, to our knowledge. The widespread application of models based on the assumption that source memory is limited to recognized items suggests that many source researchers consider this assumption valid.

Recently, researchers have begun to explore models based on the continuous retrieval of source information, as opposed to the discrete state of source identification proposed by multinomial models (e.g., Banks, 2000; DeCarlo, 2003; Glanzer, Hilford, \& Kim, 2004; Hilford, Glanzer, Kim, \& DeCarlo, 2002; Qin, Raye, Johnson, \& Mitchell, 2001; Slotnick \& Dodson, 2005; Slotnick, Klein, Dodson, $\&$ Shimamura, 2000). Figure 2 displays the equal variance signal detection model for source discrimination based on continuous evidence (Wickens, 2002). For each test item, retrieval processes yield a single evidence value on a continuum that ranges from strong evidence in favor of Source A to strong evidence in favor of Source B. Evidence values for Source A and Source B items are both normally distributed, with the mean of the Source B distribution farther along the continuum than the mean of the Source A distribution. The distance between the distribution means is measured by the parameter $d^{\prime}$, and source discriminability is determined by the degree of overlap in the distributions. To select between source alternatives, participants establish a criterion $\lambda$ for the minimum evidence value required to respond "Source B," and they respond "Source A" for evidence values that fall below this criterion.

To handle data from combined recognition and source tests, the one-dimensional (1-D) model displayed in Figure 2 must be expanded into a multivariate model (Banks, 2000; DeCarlo, 2003; Glanzer et al., 2004; Hilford et al., 2002; Slotnick \& Dodson, 2005). The multivariate model depicted in Figure 3 includes three bivariate normal distributions to represent Source A, Source B, and new items. One dimension of the model represents the discriminability of studied items from new items. On this dimension, Source A and Source B items tend to have higher evidence values than new items, and evidence values for all three item types are normally distributed. The other dimension of the model represents source evidence. On this dimension, Source B items tend to have higher evidence values

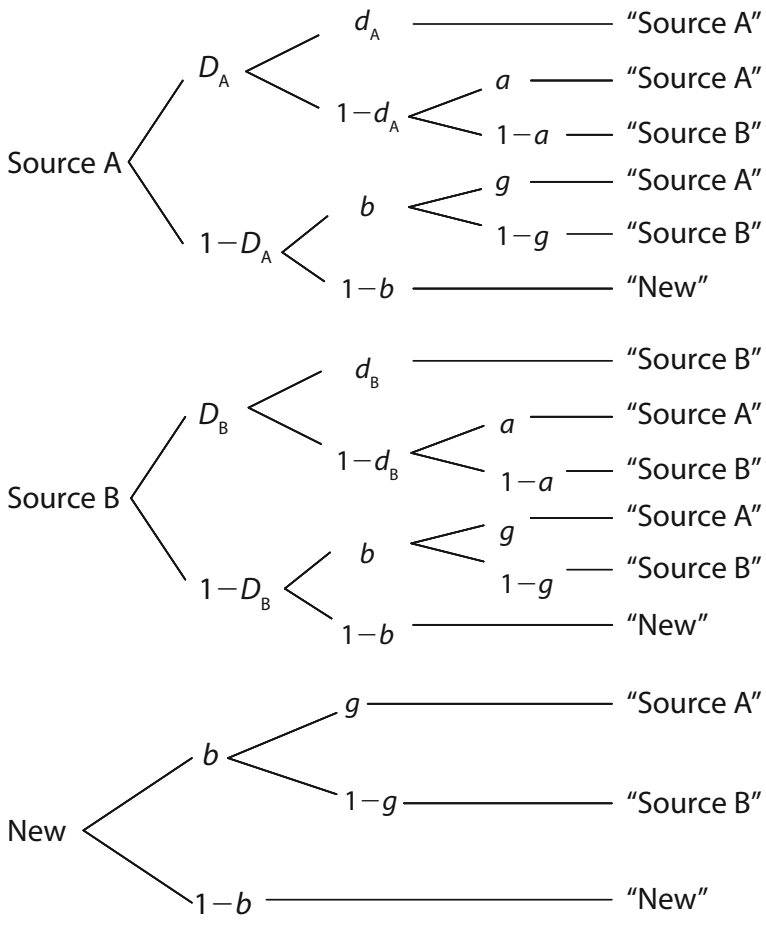

Figure 1. Example of a multinomial model for recognition/ source memory. Labels on the left refer to the actual source of the items; labels on the right refer to the source claims made by participants.

than Source A items, and both of these item types are again normally distributed. New items also have a normal distribution of source evidence values, and the new item distribution falls between the Source A and Source B distributions (i.e., new items are not clearly associated with either source). In the multivariate model, the memory evidence for each test item is represented by a point $(x, y)$ where $x$ is the item's value on the recognition evidence continuum and $y$ is the item's value on the source evidence continuum.

In Figure 3, recognition evidence is displayed on the horizontal dimension and source evidence is displayed on

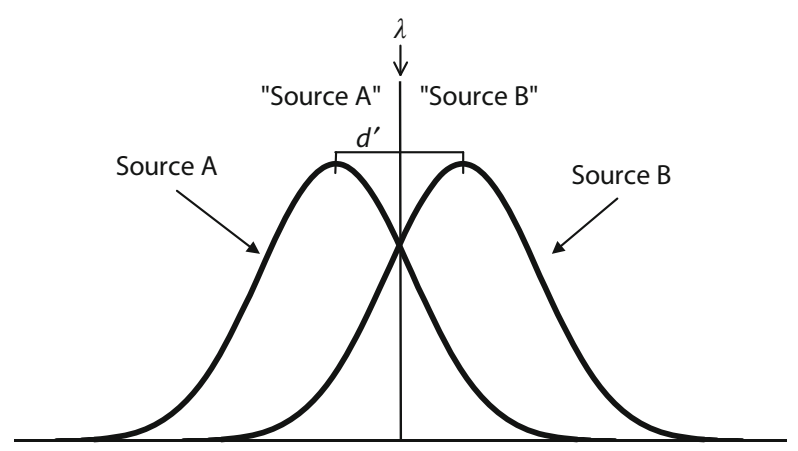

Figure 2. Signal detection model for source discrimination. The distributions represent evidence values for items from each source. The vertical line represents the response criterion, and the source labels in quotes refer to the source claims made by participants. 


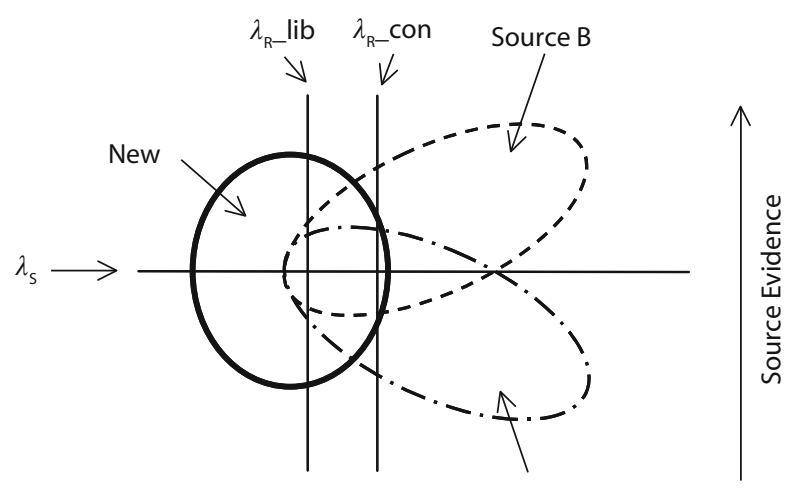

Source A

Recognition Evidence

Figure 3. The multivariate signal detection model for performance on a recognition/source test. The ovals are horizontal cross sections through the 3-D evidence distributions for each type of test item. The vertical lines represent recognition criteria and the horizontal line represents the source criterion.

the vertical dimension. The contours represent distributions for each item type. If it could be displayed, the density of evidence values from each distribution at each point $(x, y)$ would be represented as height above the page (for a 3-D depiction of the model, see Slotnick \& Dodson, 2005). Thus, the contours can be thought of as horizontal slices through the 3-D distributions (Ashby \& Maddox, 1990). Each distribution is centered on a point at the mean of the evidence on the recognition dimension and the mean of the evidence on the source dimension, and each distribution has standard deviations in both the recognition and source dimensions. The form of each distribution also depends on the correlation between evidence values on the source and recognition dimensions, $\rho$. In fitting the multivariate signal detection model to several data sets, DeCarlo (2003) found that $\rho$ was near zero for new items, but was large and had opposite signs for Source A and Source B items. $\rho$ for Source A was negative in all of the model fits, indicating that Source A items with high values on the recognition dimension also tend to have low values on the source dimension (i.e., source evidence strongly favors Source A). $\rho$ for Source B items was positive in all of the model fits, indicating that Source B items with high values on the recognition dimension also tend to have high values on the source dimension (i.e., source evidence strongly favors Source B). These correlations are reflected in the downward slant of the Source A distribution and the upward slant of the Source B distribution as recognition evidence increases in Figure 3. Psychologically, the correlation indicates that encoding and retrieval procedures that promote effective recognition memory also tend to promote effective source memory (Glanzer et al., 2004).

The multivariate model incorporates criteria along both evidence dimensions to translate evidence values into responses on a recognition/source test. The vertical lines in Figure 3 mark possible positions for the recognition criterion $\lambda_{R}$. Items to the right of $\lambda_{R}$ on the recognition dimen- sion are called "old," and items to the left of $\lambda_{\mathrm{R}}$ are called "new." $\lambda_{\mathrm{R}}$ lib shows a liberal position for the recognition criterion, and $\lambda_{\mathrm{R}}$ con shows a conservative position. The horizontal line represents the source criterion $\lambda_{\mathrm{S}}$. Items with values above $\lambda_{\mathrm{S}}$ on the source dimension are called "Source B," and items below $\lambda_{\mathrm{S}}$ are called "Source A."

The multivariate signal detection model's predictions regarding source memory for unrecognized items can be seen by evaluating the portion of the Source A and Source B distributions that fall to the left of $\lambda_{\mathrm{R}}$. When the recognition criterion is liberal $\left(\lambda_{\mathrm{R}}\right.$ lib), only a small proportion of the source distributions fall behind the criterion, and the Source A and Source B distributions almost completely overlap in this region. As a result of the extreme overlap in the source distributions, source performance should be very poor, and possibly no better than chance responding. When the recognition criterion is conservative $\left(\lambda_{\mathrm{R}}\right.$ con $)$, a larger proportion of the source distributions fall to the left of the recognition criterion. The source distributions have increasingly less overlap as recognition evidence increases; thus, including items with more recognition evidence in the "unrecognized" category should lead to higher levels of source memory for unrecognized items. In other words, source memory for unrecognized items should be above chance levels if participants are sufficiently conservative in their recognition responding. ${ }^{1}$

In each experiment reported herein, participants first made recognition decisions and then were asked to specify the source of all studied words regardless of the outcome of the recognition test. In the following experiments, we manipulated response bias on the recognition test by instructing participants that either $25 \%$ or $75 \%$ of the test words were studied. The result of primary interest was the level of source performance for studied words that were called "new" on the recognition test. If recognition memory must accompany source retrieval, then source discrimination should be at chance levels for items labeled "new." Moreover, source performance should not change on the basis of the bias manipulation. In contrast, the multivariate signal detection model predicts that participants should be able to discriminate sources for unrecognized items if the recognition criterion is sufficiently conservative (see Figure 3). Thus, this model predicts abovechance levels of source memory for unrecognized items when recognition responding is conservative. When recognition responding is liberal, participants should display very poor source discrimination for unrecognized items, perhaps not exceeding chance levels.

\section{EXPERIMENT 1}

In this experiment, participants studied words that appeared in either a large or a small font size. Participants completed a recognition test given instructions that either $25 \%$ or $75 \%$ of the words on the test were studied. In fact, $50 \%$ of the test words were studied for both instruction groups. Following the recognition test, participants were re-presented with all of the studied words and were asked to decide whether each word appeared in a large or small font size in the study phase. 


\section{Method}

Participants. A total of 98 Louisiana State University undergraduates participated to earn extra credit in their psychology courses. Participants were randomly assigned to the proportion instruction conditions, with 51 participants in the $25 \%$ condition and 47 participants in the $75 \%$ condition.

Design and Materials. This experiment conformed to a 2 (source: large or small) $\times 2$ (proportion instruction: $25 \%$ targets or $75 \%$ targets) factorial design with source manipulated within participants and proportion instruction manipulated between participants. Again, the proportion variable refers only to information reported in the test instructions. The recognition test for all participants was actually half targets and half lures. We selected a list of 148 words from the MRC psycholinguistic database (Coltheart, 1981) for use in our experiments. All of the words were four- to six-letter nouns with familiarity and concreteness ratings between 350 and 600 (on scales ranging from 100 to 700). Eight of the words were used as fillers, with 4 appearing at the very beginning of the study list and 4 appearing at the very end. Of the remaining 140 words, 35 were studied in a large font, 35 were studied in a small font, and 70 were used as lures on the recognition test. The words assigned to each item class were counterbalanced across participants. Words on the encoding list appeared in Comic Sans MS font. Large words appeared in 70-point font and small words appeared in 14-point font. The first (recognition) test contained the 70 studied words along with 70 unstudied items. The second (source) test contained only the 70 studied words. Words appeared in 36-point Courier New font on both tests; thus, the appearance of the test words was clearly distinct from the appearance of the words at encoding in terms of both font size and font style. We used distinct fonts so that participants would not confuse an item's appearance on the recognition test with the item's appearance in the study phase.

Procedure. Before the study phase, participants were informed that they would need to remember the words on the study list for a memory test, but they were not told to remember the size of the words. Words appeared in a random order at encoding. Each word remained on the screen for $1,800 \mathrm{msec}$, followed by $200 \mathrm{msec}$ in which the screen was blank. After all of the words were presented, instructions for the distractor task appeared on the screen. For this task, scrambled pictures appeared on the screen and participants were required to rearrange the components until the picture was unscrambled. Participants worked on the picture task for $3 \mathrm{~min}$, and then the recognition test instructions appeared. Participants were told that they would see words from the study list mixed in with unstudied items. Participants were told that either $25 \%$ or $75 \%$ of the test words were actually studied, depending on their assigned condition. For each word, participants indicated whether or not the word appeared in the study phase by pressing a $Y$ sticker placed on the "D" key or an $N$ sticker placed on the "J" key. Participants worked through the recognition test at their own pace, and then the source test instructions appeared on the screen. Participants were informed that they would see all of the words from the study phase, and that they would indicate whether each word appeared in a large or small font by pressing either an $L$ sticker placed on the "C" key or an $S$ sticker placed on the "N" key. Participants once again worked through the test trials at their own pace.

\section{Results and Discussion}

We used an alpha of .05 as the criterion of significance in all inferential tests. The recognition hit and false alarm results of Experiment 1 are reported in the first row of Table 1. Hit rates for both large (reported as Source A) and small (reported as Source B) targets were higher in the $75 \%$ than in the $25 \%$ proportion instruction condition, and the large hit rate was slightly higher than the small hit rate. The proportion instruction manipulation also led to an increase in false alarm rate from the $25 \%$ to the $75 \%$ condition. The fact that participants made more positive recognition responses for both targets and lures when they were told that the test contained a higher proportion of studied items demonstrates the effectiveness of our bias manipulation.

We also computed signal detection measures of recognition performance (see Table 2), and we focus on these measures for our inferential analyses. The $d_{\mathrm{R}}$ measure represents the distance of the Source A or Source B target distributions from the lure distribution on the recognition dimension, and is calculated by the formula $d_{\mathrm{R}}=\sigma z(\mathrm{HR})-z(\mathrm{FAR})$, where $z$ is the inverse of the standard Gaussian cumulative distribution function, $\sigma$ is the standard deviation of the target distribution relative to that of the lure distribution, HR is the hit rate, and FAR is the false alarm rate (we adjusted the hit and false alarm rates to eliminate values of 0 or 1 as in Snodgrass and Corwin [1988]). Previous recognition studies have established that the target distribution is more variable than the lure distribution (e.g., Glanzer, Kim, Hilford, \& Adams, 1999). More specifically, the target distribution typically has a standard deviation around 1.25 times the standard deviation of the lure distribution, a finding that is consistent across a variety of experimental manipulations (Ratcliff, McKoon, \& Tindall, 1994; Ratcliff, Sheu, \& Gronlund, 1992). Therefore, our $d_{\mathrm{R}}$ computations assume $\sigma=1.25$. We also report $\lambda_{R}$, the distance of the response criterion from the mean of the lure distribution on the recognition dimension calculated by the formula $\lambda_{\mathrm{R}}=z(1-\mathrm{FAR})$.

A 2 (source) $\times 2$ (proportion instruction) ANOVA on the $d_{\mathrm{R}}$ measures revealed a main effect of source $[F(1,96)=$ $\left.9.80, M S_{\mathrm{e}}=.118, p<.01\right]$, such that evidence was more discriminable for large than for small items. There was no main effect of proportion instruction and no interaction

Table 1

Recognition Performance in the $25 \%$ and $75 \%$ Proportion Instruction Conditions of Experiments 1-3

\begin{tabular}{|c|c|c|c|c|c|c|c|c|c|c|c|c|}
\hline \multirow[b]{4}{*}{ Experiment } & \multicolumn{12}{|c|}{ Recognition Measure and Proportion } \\
\hline & \multicolumn{4}{|c|}{ Source A HR } & \multicolumn{4}{|c|}{ Source B HR } & \multicolumn{4}{|c|}{ FAR } \\
\hline & \multicolumn{2}{|c|}{$25 \%$} & \multicolumn{2}{|c|}{$75 \%$} & \multicolumn{2}{|c|}{$25 \%$} & \multicolumn{2}{|c|}{$75 \%$} & \multicolumn{2}{|c|}{$25 \%$} & \multicolumn{2}{|c|}{$75 \%$} \\
\hline & $M$ & $\overline{S E}$ & $M$ & $\overline{S E}$ & $M$ & $\overline{S E}$ & $M$ & $\overline{S E}$ & $M$ & $\overline{S E}$ & $M$ & $\overline{S E}$ \\
\hline 1 & .61 & .02 & .79 & .02 & .56 & .02 & .76 & .02 & .21 & .02 & .45 & .02 \\
\hline 2 & .84 & .02 & .91 & .02 & .83 & .02 & .89 & .02 & .09 & .01 & .23 & .03 \\
\hline 3 & .76 & .04 & .89 & .02 & .75 & .03 & .88 & .02 & .05 & .01 & .11 & .03 \\
\hline
\end{tabular}

Note-HR, hit rate; FAR, false alarm rate. For Experiment 1, "Source A" refers to large items and "Source B" refers to small items. For Experiments 2 and 3, "Source A" refers to pleasantness items and "Source B" refers to imageability items. 
Table 2

Recognition Signal Detection Measures for Experiments 1-3

\begin{tabular}{|c|c|c|c|c|c|c|}
\hline \multirow{3}{*}{$\begin{array}{l}\text { Experiment and } \\
\text { Instruction Condition }\end{array}$} & \multicolumn{6}{|c|}{ Signal Detection Measure } \\
\hline & \multicolumn{2}{|c|}{$d_{\mathrm{R}}-\operatorname{Src} \mathrm{A}$} & \multicolumn{2}{|c|}{$d_{\mathrm{R}}-\operatorname{Src~B}$} & \multicolumn{2}{|c|}{$\lambda_{\mathrm{R}}$} \\
\hline & $M$ & $S E$ & $M$ & $S E$ & $M$ & $S E$ \\
\hline \multicolumn{7}{|l|}{ Experiment 1} \\
\hline $25 \%$ & 1.26 & 0.08 & 1.08 & 0.08 & 0.88 & 0.07 \\
\hline $75 \%$ & 1.22 & 0.09 & 1.08 & 0.09 & 0.15 & 0.07 \\
\hline \multicolumn{7}{|l|}{ Experiment 2} \\
\hline $25 \%$ & 2.76 & 0.14 & 2.74 & 0.14 & 1.43 & 0.09 \\
\hline $75 \%$ & 2.65 & 0.14 & 2.49 & 0.14 & 0.84 & 0.11 \\
\hline \multicolumn{7}{|l|}{ Experiment 3} \\
\hline $25 \%$ & 2.65 & 0.16 & 2.63 & 0.16 & 1.74 & 0.07 \\
\hline $75 \%$ & 3.00 & 0.17 & 2.96 & 0.16 & 1.38 & 0.12 \\
\hline
\end{tabular}

Note-For Experiment 1, "Src A" refers to large items and "Src B" refers to small items. For Experiments 2 and 3, "Src A" refers to pleasantness items and "Src B" refers to imageability items.

$\left[F(1,96)=0.03, M S_{\mathrm{e}}=.602\right.$, n.s., and $F(1,96)=0.13$, $M S_{\mathrm{e}}=.118$, n.s., respectively]. $\lambda_{\mathrm{R}}$ was significantly higher in the $25 \%$ target condition than in the $75 \%$ target condition $[t(96)=7.52, p<.001]$. These results show that the proportion instruction manipulation did not have an impact on recognition discriminability, but altered the amount of evidence that participants required to say "old."

We measured source memory as the proportion of target words that were attributed to the correct source. We evaluated proportion correct both across all targets and conditionalized on "no" responses on the recognition test. We tested for source discrimination by comparing source scores to a value of .50 (chance performance). We also computed source discriminability, $d_{\mathrm{S}}$, which is the distance between the means of the Source A and Source B distributions on the source dimension. $d_{\mathrm{S}}$ was computed with the same formula as $d_{\mathrm{R}}$, with the proportion of Source 1 items called "Source 1" as the hit rate and the proportion of Source 2 items called "Source 1" as the false alarm rate (we also applied the Snodgrass \& Corwin [1988] adjustment to these values). In contrast to recognition, source studies commonly find that the Source 1 and Source 2 distributions have roughly equal standard deviations (see a review in Hilford et al., 2002). Therefore, we based our $d_{\mathrm{S}}$ calculations on an equal variance model $(\sigma=1)$.

The proportion of correct source attributions for this experiment is reported in the first row of Table 3 , and the $d_{\mathrm{S}}$ scores are reported in Table 4 along with the source hit and false alarm rates. When computed across all items, regardless of recognition response, source discriminability $\left(d_{\mathrm{S}}\right)$

Table 3

Proportions of Correct Source Attributions for All Target Items and for Unrecognized Targets in the $25 \%$ and $75 \%$ Conditions of Experiments 1-3

\begin{tabular}{|c|c|c|c|c|c|c|c|c|}
\hline \multirow[b]{4}{*}{ Experiment } & \multicolumn{8}{|c|}{ Analyzed Items and Proportion Instruction } \\
\hline & \multicolumn{4}{|c|}{ All Targets } & \multicolumn{4}{|c|}{ Unrecognized Targets } \\
\hline & \multicolumn{2}{|c|}{$25 \%$} & \multicolumn{2}{|c|}{$75 \%$} & \multicolumn{2}{|c|}{$25 \%$} & \multicolumn{2}{|c|}{$75 \%$} \\
\hline & $M$ & $S E$ & $M$ & $S E$ & $M$ & $S E$ & $M$ & $S E$ \\
\hline 1 & .56 & .01 & .56 & .01 & .54 & .01 & .50 & .02 \\
\hline 2 & .70 & .02 & .69 & .03 & .62 & .04 & .51 & .05 \\
\hline 3 & .73 & .02 & .76 & .02 & .67 & .05 & .53 & .04 \\
\hline
\end{tabular}

did not differ on the basis of the instruction manipulation $[t(96)=0.47$, n.s. $]$, which demonstrates that random assignment successfully equated overall source performance across the groups. The data of greatest interest were the source scores for targets that participants failed to recognize on the first test. The proportion of correct source attributions for unrecognized items was significantly above chance performance in the $25 \%$ condition $[t(50)=3.72$, $p<.001]$, but not in the $75 \%$ condition $[t(46)=0.01$, n.s.]. The multivariate signal detection model predicts better source memory for unrecognized items in the $25 \%$ than in the $75 \%$ conditions, so we compared these conditions with a directional $t$ test. The test confirmed the advantage for the $25 \%$ condition $[t(96)=1.81, p<.05] .^{2}$

The results showed evidence of source memory for unrecognized items when participants responded conservatively on the recognition test, but not when participants responded liberally. This pattern is consistent with the predictions of the multivariate signal detection model. Although source scores were significantly higher than .50 for unrecognized items in the $25 \%$ target condition, source performance was very poor for these items (only .54 were attributed to the correct source). This is not surprising, considering that source performance was poor in general, regardless of the recognition claim (only $56 \%$ of all targets were attributed to the correct source). In the following experiment, we attempted to increase the overall level of source performance to discover whether higher overall source memory translates into higher levels of source memory for unrecognized items. We did so by replacing the large/small sources with pleasantness and imageability ratings.

\section{EXPERIMENT 2}

\section{Method}

Participants. A total of 69 Louisiana State University undergraduates participated in exchange for extra credit in their psychology courses. Participants were randomly assigned to the proportion instruction conditions, with 34 in the $25 \%$ condition and 35 in the $75 \%$ condition.

Design, Materials, and Procedure. All of the methodological details matched those of Experiment 1, with two exceptions. First, we used pleasantness and imageability ratings as sources instead of large and small font sizes (all words appeared in 18-point font). For half of the words on the study list, participants were asked to rate how pleasant the word was to them on a scale from 1 (very unpleasant) to 5 (very pleasant). For the remaining words, participants rated how easy it was for them to imagine a referent of the word on a scale from 1 (very difficult) to 5 (very easy). All study words remained on the computer screen until a rating was made. At test, the source response keys were labeled with $P$ and $I$ stickers. Second, we added 52 filler words to the original 8 , resulting in a study list that began with 30 fillers and ended with 30 fillers. We added extra filler words to increase the length of the study list in an attempt to decrease recognition performance. Prior research in our laboratory indicated that response bias manipulations were less effective when recognition performance was very high.

\section{Results and Discussion}

The hit and false alarm rates for this experiment appear in the second row of Table 1. As in the first experiment, the false alarm rate was higher in the $75 \%$ condition than in the $25 \%$ condition. Hit rates for both pleasantness (Source A) and imageability (Source B) targets were also higher in the 
Table 4

Source Discriminability for Experiments 1-3

\begin{tabular}{|c|c|c|c|c|c|c|}
\hline \multirow{3}{*}{$\begin{array}{l}\text { Experiment and } \\
\text { Instruction Condition }\end{array}$} & \multicolumn{6}{|c|}{ Memory Measure } \\
\hline & \multicolumn{2}{|c|}{$\begin{array}{l}\text { Source } \\
\text { HR }\end{array}$} & \multicolumn{2}{|c|}{$\begin{array}{l}\text { Source } \\
\text { FAR }\end{array}$} & \multicolumn{2}{|c|}{$d_{\mathrm{S}}$} \\
\hline & $M$ & $S E$ & $M$ & $S E$ & $M$ & $S E$ \\
\hline \multicolumn{7}{|l|}{ Experiment 1} \\
\hline $25 \%$ & .44 & .02 & .32 & .02 & 0.36 & 0.07 \\
\hline $75 \%$ & .47 & .02 & .36 & .02 & 0.30 & 0.05 \\
\hline \multicolumn{7}{|l|}{ Experiment 2} \\
\hline $25 \%$ & .65 & .03 & .26 & .02 & 1.10 & 0.12 \\
\hline $75 \%$ & .67 & .03 & .30 & .03 & 1.07 & 0.16 \\
\hline \multicolumn{7}{|l|}{ Experiment 3} \\
\hline $25 \%$ & .72 & .03 & .27 & .03 & 1.23 & 0.14 \\
\hline $75 \%$ & .74 & .02 & .23 & .03 & 1.49 & 0.15 \\
\hline
\end{tabular}

Note-HR, hit rate; FAR, false alarm rate. For Experiment 1, the source hit rate refers to large items called "large" and the source false alarm rate refers to small items called "large." For Experiments 2 and 3, the source hit rate refers to pleasantness items called "pleasantness" and the source false alarm rate refers to imageability items called "pleasantness."

$75 \%$ condition. The $d_{\mathrm{R}}$ values in the second row of Table 2 did not differ on the basis of source $\left[F(1,67)=1.95, M S_{\mathrm{e}}=\right.$ .145 , n.s.] or proportion instruction $[F(1,67)=1.00$, $M S_{\mathrm{e}}=1.20$, n.s.]. The interaction was also nonsignificant $\left[F(1,67)=1.12, M S_{\mathrm{e}}=.145\right.$, n.s. $]$. The $25 \%$ target condition led to a significantly higher response criterion $\left(\lambda_{\mathrm{R}}\right)$ than did the $75 \%$ condition $[t(67)=4.32, p<.001]$. Thus, once again, the proportion instruction manipulation did not have an impact on recognition discriminability but successfully altered the amount of evidence that participants required to call an item "old."

Table 3 reveals that the overall source scores were considerably higher than those from Experiment 1; thus, our attempt to increase source memory was successful. The $d_{\mathrm{S}}$ values in Table 4 also exceed those from Experiment 1, and these measures did not significantly differ on the basis of the proportion instruction conditions $[t(67)=0.12$, n.s. $]$. The proportion of correct attributions for unrecognized items was significantly above chance in the $25 \%$ condition $[t(32)=3.27, p<.01]$ but not in the $75 \%$ condition $[t(33)=0.11$, n.s. $] .{ }^{3}$ Moreover, unrecognized source accuracy was significantly higher in the $25 \%$ condition than in the $75 \%$ condition $[t(65)=1.77, p<.05$, one-tailed $]$. The critical result of the first experiment was replicated, in that participants displayed above-chance levels of source accuracy for unrecognized items only following conservative responding on the recognition test. Comparing unrecognized proportion correct in the $25 \%$ condition to the same condition of Experiment 1 reveals that increasing the overall level of source memory also increased source memory for unrecognized items. Indeed, the level of source memory for unrecognized items following conservative recognition responding in Experiment 2 exceeded the level of overall source memory from Experiment 1.

\section{EXPERIMENT 3}

The results of Experiments 1 and 2 converge on the conclusion that participants can specify the source of unrecognized items when they apply a conservative recogni- tion criterion. However, the fact that participants made recognition and source judgments on separate tests may introduce an interpretive difficulty. Specifically, one could claim that some words that were not recognized on the first test were nevertheless recognized when they appeared on the second test, in a type of reminiscence effect (Payne, 1987). We have concluded that our results demonstrate accurate source memory for unrecognized items, but the results may in truth demonstrate accurate source memory for items recognized on the second test but not the first. Although we consider this possibility unlikely, we acknowledge that demonstrating accurate source memory immediately following a failed attempt at recognition would provide the most compelling evidence for our claims. In Experiment 3, we tested recognition and source memory on the same test. Participants made a source decision for each studied item immediately following their recognition decision for that item.

Another possible criticism of the first two experiments is that participants artificially altered their recognition responses in order to better match the target probability mentioned in the instructions. For example, if participants attempted to say "yes" only $25 \%$ of the time when informed that $25 \%$ of the test items were studied, then they may have had to say "no" to words that they actually recognized to keep their proportion of "yes" responses low enough. Thus, we may have observed not source memory for unrecognized items, but source memory for recognized items given "no" responses, in an attempt to match the reported probability of targets. We investigated this possibility by manipulating the actual proportion of targets on the test along with the proportion reported to participants. With very few targets on the test in the $25 \%$ condition, participants will not have to artificially decline recognized test candidates in order to lower their proportion of "yes" responses.

\section{Method}

Participants. A total of 67 Louisiana State University undergraduates participated to earn extra credit in their psychology courses, with 34 randomly assigned to the $25 \%$ proportion instruction condition and 33 assigned to the $75 \%$ condition.

Design, Materials, and Procedure. The methodological details matched those of Experiment 2, except that we manipulated the actual proportion of targets on the test, and source decisions were made immediately after recognition decisions on the first test. For participants in the $25 \%$ instruction condition, the test contained 10 targets from the pleasantness source, 10 targets from the imageability source, and 70 lures. Thus, the test was $22 \%$ studied items. Targets from both sources were taken from randomly selected study list positions. For participants in the $75 \%$ instruction condition, the test contained all 35 targets from both the pleasantness and imageability sources, along with 20 lures. Thus, the test was $78 \%$ studied items. For consistency with the previous experiments, we will refer to the conditions by the approximate proportion mentioned in the instructions and not the actual proportion of targets. Prior to the test, participants were given the same recognition and source instructions as in the other experiments, except that they were informed that they would be making both judgments on the same test. They were told that they would be asked to indicate the source of each test word that they claimed to recognize and all studied words, regardless of their recognition claim. That is, participants made source claims for all targets and also for lures that they claimed were old. 


\section{Results and Discussion}

Hit rates were higher in the $75 \%$ condition than in the $25 \%$ condition, as was the false alarm rate. $d_{\mathrm{R}}$ scores did not vary on the basis of target source $[F(1,65)=0.13$, $M S_{\mathrm{e}}=.278$, n.s.], instruction condition $[F(1,65)=2.64$, $M S_{\mathrm{e}}=1.466$, n.s. $]$, or their interaction $[F(1,65)=0.01$, n.s.]. $\lambda_{\mathrm{R}}$ was higher in the $25 \%$ condition than in the $75 \%$ condition $[t(65)=2.59, p<.05]$. Thus, the recognition results again indicate that the proportion manipulation altered participants' recognition criterion without significantly affecting their memory for the studied items.

Overall source discriminability $\left(d_{\mathrm{S}}\right)$ did not differ on the basis of instruction condition $[t(65)=1.28, n . s$.$] . As in$ the previous experiments, participants attributed a higher proportion of unrecognized targets to the correct source in the $25 \%$ condition than in the $75 \%$ condition $[t(59)=$ $2.17, p<.05$, one-tailed]. Source scores for unrecognized items exceeded chance performance in the $25 \%$ condition $[t(29)=3.56, p<.001]$, but not in the $75 \%$ condition $[t(30)=0.71$, n.s. $]$.

Results once again indicated that participants could accurately specify the source of unrecognized items when they were conservative in their recognition decisions. The results also demonstrate that this phenomenon can be observed even when source queries immediately follow failed recognition attempts. Moreover, the results of the previous experiments were replicated even though the proportion of targets mentioned in the instructions closely matched the actual target proportion. Therefore, we are confident that our results do not reflect a strategy whereby participants reject recognized targets in an attempt to match their proportion of "yes" responses to the reported target proportion.

\section{GENERAL DISCUSSION}

All three experiments revealed evidence that participants have the ability to specify the source of unrecognized items. Source accuracy for unrecognized items exceeded chance levels in each of the $25 \%$ proportion instruction conditions. As noted in the introduction, standard test procedures reflect the assumption that source memory is only possible for recognized items, and this assumption has also guided the application of multinomial models to source data. Our results refute this assumption.

The multivariate signal detection model successfully predicted the qualitative pattern of findings from each of our experiments. In situations in which recognition and source evidence are positively correlated (DeCarlo, 2003), the multivariate model predicts that participants will be able to specify the source of unrecognized items if the recognition criterion is set at a sufficiently conservative value (see Figure 3). Thus, this model explains not only the presence of source memory for unrecognized items, but also the fact that participants showed no ability to specify the source of unrecognized items when we induced liberal recognition responding (i.e., in the $75 \%$ proportion instruction conditions).

In general, our results are consistent with the view that memory retrieval involves the continuous accumulation of evidence. For each test candidate on a recognition/source test, participants retrieve an amount of evidence that the item was on the study list and an amount of evidence that the item was seen in a particular source versus the alternative source. Even if the recognition evidence fails to pass the recognition criterion, the source evidence may still favor the correct source. Moreover, sources can be discriminable even if participants do not vividly remember the exact source characteristics of the test candidate (see Hicks, Marsh, \& Ritschel, 2002). Consistent with this conclusion, Dodson et al. (1998) demonstrated that source decisions can be based on "partial" source evidence, in the absence of memory for exact source characteristics. In that study, participants heard words from four voices, two of which were male and two of which were female. Results showed that even when participants could not remember the exact voice that spoke a test candidate, they were likely to attribute the word to a speaker of the same gender as the correct speaker. Thus, even when source evidence was not sufficiently differentiated to specify the correct voice, participants often experienced a degree of source evidence that was adequate to specify the gender of the voice. Our finding of source discrimination for items that were not remembered vividly enough to pass a conservative recognition criterion provides further evidence that source decisions can be based on incomplete source information. A recent study by Cook, Marsh, and Hicks (2006) bolsters this conclusion by demonstrating source discrimination for target words that could not be produced on a cued recall test.

Although our results seem most consistent with continuous evidence, models assuming discrete retrieval could potentially be altered to successfully capture our findings. Existing multinomial models reflect the assumption that source discrimination can only follow successful recognition, but future models may abandon this assumption. For example, models could posit a memory state that is intermediate between complete detection and guessing, with this state representing the recovery of partial item information. ${ }^{4}$ If one assumes that source details are available for partial item memories, and that partial item memories are labeled "old" under liberal responding and "new" under conservative responding, then multinomial models could potentially accommodate our results. Specifically, such a model would explain how items in the unrecognized category could support source discrimination when recognition responding is conservative. However, this example demonstrates that multinomial models would need to be altered post hoc to accommodate a result that the multivariate signal detection model naturally predicted.

Researchers commonly test models by evaluating their ability to fit empirical data. An exclusive reliance on fit as an index of model quality may be ill advised, because models can often closely fit data produced by processes other than the ones assumed by the model (Myung, 2000), and accurate models may fail to successfully fit data that are compromised by contaminant processes (see, e.g., Ratcliff \& Tuerlinckx, 2002). For these reasons, assessing a model's predictive ability is an important supplement to fit assessments (Smith \& Duncan, 2004). We showed that the 
multivariate signal detection model generates predictions for source discrimination in the absence of recognition, and that these predictions contradict an assumption reflected in both standard source test procedures and existing multinomial models of source memory. The results of three experiments adhered to the signal detection model's predictions and refuted the assumption that source discrimination is only possible for recognized items. In conjunction with model fits such as those reported by DeCarlo (2003), the ability of the multivariate signal detection model to make successful predictions suggests that this model will be a valuable tool in source memory research.

\section{AUTHOR NOTE}

We thank Alicia Hymel, Lauren Robinson, Kristen Abbondonte, Kimberly Mancina, and Christine Vinci for their dedicated help in collecting the data. Correspondence concerning this article should be addressed to J. L. Hicks, Department of Psychology, Louisiana State University, Baton Rouge, LA 70803-5501 (e-mail: jhicks@1su.edu).

\section{REFERENCES}

Ashby, F. G., \& Maddox, W. T. (1990). Integrating information from separable psychological dimensions. Journal of Experimental Psychology: Human Perception \& Performance, 16, 598-612.

BANKs, W. P. (2000). Recognition and source memory as multivariate decision processes. Psychological Science, 11, 267-273.

BAtchelder, W. H., \& Riefer, D. M. (1990). Multinomial processing models of source monitoring. Psychological Review, 97, 548-564.

Bayen, U. J., Murnane, K., \& Erdfelder, E. (1996). Source discrimination, item detection, and multinomial models of source monitoring. Journal of Experimental Psychology: Learning, Memory, \& Cognition, 22, 197-215.

Coltheart, M. (1981). The MRC psycholinguistic database. Quarterly Journal of Experimental Psychology, 33A, 497-505.

CoOK, G. I., MARSH, R. L., \& HICKS, J. L. (2006). Source memory in the absence of successful cued recall. Journal of Experimental Psychology: Learning, Memory, \& Cognition, 32, 828-835.

DeCarlo, L. T. (2003). Source monitoring and multivariate signal detection theory, with a model for selection. Journal of Mathematical Psychology, 47, 292-303.

Dodson, C. S., Holland, P. W., \& Shimamura, A. P. (1998). On the recollection of specific- and partial-source information. Journal of Experimental Psychology: Learning, Memory, \& Cognition, 24, 1121-1136.

Dodson, C. S., \& Shimamura, A. P. (2000). Differential effects of cue dependency on item and source memory. Journal of Experimental Psychology: Learning, Memory, \& Cognition, 26, 1023-1044.

Glanzer, M., Hilford, A., \& KIM, K. (2004). Six regularities of source recognition. Journal of Experimental Psychology: Learning, Memory, \& Cognition, 30, 1176-1195.

Glanzer, M., Kim, K., Hilford, A., \& Adams, J. K. (1999). Slope of the receiver-operating characteristic in recognition memory. Journal of Experimental Psychology: Learning, Memory, \& Cognition, 25, 500-513.

Hicks, J. L., MARSh, R. L., \& Ritschel, L. (2002). The role of recollection and partial information in source monitoring. Journal of Experimental Psychology: Learning, Memory, \& Cognition, 28, 503-508.

Hilford, A., Glanzer, M., Kim, K., \& DeCarlo, L. T. (2002). Regularities of source recognition: ROC analysis. Journal of Experimental Psychology: General, 131, 494-510.
MeISER, T., \& BRöDER, A. (2002). Memory for multidimensional source information. Journal of Experimental Psychology: Learning, Memory, \& Cognition, 28, 116-137.

MyUnG, I. J. (2000). The importance of complexity in model selection. Journal of Mathematical Psychology, 44, 190-204.

PAYNE, D. G. (1987). Hypermnesia and reminiscence in recall: A historical and empirical review. Psychological Bulletin, 101, 5-27.

Qin, J., RaYe, C. L., Johnson, M. K., \& Mitchell, K. J. (2001). Source ROCs are (typically) curvilinear: Comment on Yonelinas (1999). Journal of Experimental Psychology: Learning, Memory, \& Cognition, 27, 1110-1115.

Ratcliff, R., McKoon, G., \& Tindall, M. (1994). Empirical generality of data from recognition memory receiver-operating characteristic functions and implications for the global memory models. Journal of Experimental Psychology: Learning, Memory, \& Cognition, 20, 763-785.

Ratcliff, R., Sheu, C.-F., \& Gronlund, S. D. (1992). Testing global memory models using ROC curves. Psychological Review, 99, 518-535.

RatClifF, R., \& Tuerlinckx, F. (2002). Estimating parameters of the diffusion model: Approaches to dealing with contaminant reaction times and parameter variability. Psychonomic Bulletin \& Review, 9, 438-481.

SLotNick, S. D., \& Dodson, C. S. (2005). Support for a continuous (single-process) model of recognition memory and source memory. Memory \& Cognition, 33, 151-170.

Slotnick, S. D., Klein, S. A., Dodson, C. S., \& Shimamura, A. P. (2000). An analysis of signal detection and threshold models of source memory. Journal of Experimental Psychology: Learning, Memory, \& Cognition, 26, 1499-1517.

Smith, D. G., \& Duncan, M. J. J. (2004). Testing theories of recognition memory by predicting performance across paradigms. Journal of Experimental Psychology: Learning, Memory, \& Cognition, 30, 615-625.

Snodgrass, J. G., \& Corwin, J. (1988). Pragmatics of measuring recognition memory: Applications to dementia and amnesia. Journal of Experimental Psychology: General, 117, 34-50.

Wickens, T. D. (2002). Elementary signal detection theory. New York: Oxford University Press.

\section{NOTES}

1. With no correlation between recognition and source evidence, the model predicts that discriminability for unrecognized items should equal overall source discriminability (i.e., for recognized and unrecognized items) and should not change on the basis of the position of the recognition criterion. Previous research strongly suggests that recognition and source evidence values are correlated (DeCarlo, 2003; Slotnick \& Dodson, 2005; Slotnick et al., 2000), so we do not seriously consider the no-correlation prediction.

2. We do not report $d_{\mathrm{S}}$ measures conditional on failed recognition because a large number of participants had undefined values resulting from proportions of 0 or 1 . Moreover, the number of unrecognized items varied across conditions, so the Snodgrass and Corwin (1988) correction would have a differential impact on $d_{\mathrm{S}}$ from different conditions.

3. One participant from each proportion instruction condition recognized all target items and could not be included in the unrecognized item analyses.

4. We thank David Riefer for suggesting this possibility.

(Manuscript received October 11, 2006; revision accepted for publication July 15,2007 .) 